\title{
RESILIENCIA EN MADRES E HIJOS CON CÁNCER
}

\author{
RESILIENCE IN MOTHERS AND CHILDREN WITH CANCER
}

\author{
Norma Ivonne González-Arratia, Dalia Nieto y José Luis Valdez \\ Facultad de Ciencias de la Conducta, Universidad Autónoma del Estado de México.
}

\section{Resumen}

El cáncer infantil plantea muchos retos compartidos durante el transcurso de la enfermedad tanto para los niños como sus familias y en especial para la madre, por lo que esta investigación tiene por objetivo identificar las características de resiliencia entre madres y sus respectivos hijos estos últimos que tienen diagnóstico de cáncer, así como conocer el grado de relación entre ambos. Se contó con la participación de 60 individuos, de los cuales 30 son niños diagnosticados con cáncer, de ambos sexos, entre 8 y 17 años de edad, y los restantes 30 participantes corresponden a sus respectivas madres, con una edad entre 28 y 60 años. Se aplicaron dos medidas de resiliencia (1) cuestionario de resiliencia para niños y (2) escala de Resiliencia (Fuerza y seguridad personal) para adultos. Los resultados indican alta resiliencia en los niños en las tres dimensiones (factores protectores internos, externos y empatía). La resiliencia materna muestra ser alta en la dimensión altruismo, seguida de un nivel de resiliencia moderada en las dimensiones de: autoestima, familia, seguridad y afiliación. Se encontró una asociación entre la resiliencia del niño (empatía) y de su madre (seguridad). Se concluye que la familia es un factor de protección indispensable para favorecer el desarrollo de la resiliencia infantil, y el rol especialmente de la madre es necesario cuando se trata de niño con cáncer. Se sugiere que los pacientes, familiares y profesionales se integren a fin de incentivar el óptimo desarrollo de niño e ir más allá de sólo el tratamiento médico.

Palabras clave: Resiliencia, cáncer infantil, madre-hijo.

\section{Abstract}

Childhood cancer poses many challenges shared during the course of the disease for children and their families, and especially for the mother, which is why this research aims identify characteristics of resiliency between mothers and their respective children these last who have cancer, as well as know the degree of relationship between the two. We had the participation of 60 individuals, of which 30 are children diagnosed with cancer, men and women, between 8 and 17 years of age, and the remaining 30 participants correspond to their respective mothers aged 28 to 60 years. (1) Questionnaire resiliency two resiliency measures were applied for children and (2) scale of resilience (force and personal safety) for adults. The results indicate high resiliency in children in three dimensions (internal, external protective factors, and empathy). The maternal resiliency shows to be high in dimension altruism, followed by a level of resiliency moderate dimensions: selfesteem, family, security, and membership. An association was found between the resilience of children (empathy) and his mother (security). It is concluded that family is essential for the development of the child resiliency protection factor, and especially the mother role is necessary when it comes to children with cancer. It is suggested that the patients, families and professionals are integrated in order to promote the optimal development of children and go beyond just the medical treatment.

Key words: Resiliency, childhood cancer, mother and child.

Correspondencia:

Norma Ivonne González-Arratia

Mariano Matamoros sur 706 Col. Francisco Murguía, C.P. 50130, Toluca, Estado de México. México.

E-mail: nigalf@yahoo.com.mx 


\section{INTRODUCCIÓN}

El estudio de la resiliencia como la capacidad de recuperarse de eventos vitales y salir fortalecido de ellos, se ha convertido en el foco de atención de las últimas décadas, ya que tradicionalmente la Psicología se ha centrado más en el déficit y el daño, que en el desarrollo de capacidades individuales ${ }^{(1)}$.

El término resiliencia surge para dar una explicación de casos en donde se ha observado que algunos seres humanos logran superar condiciones severamente adversas y que, inclusive, logran transformarlas en una ventaja o un estímulo para su desarroIlo bio-psico-social. En física, resistencia es la acción de un cuerpo contra una fuerza opuesta. Viene del verbo resilio que significa saltar hacia atrás, rebotar, repercutir. Fue adoptado por las Ciencias Sociales para caracterizar a aquellos sujetos que, a pesar de nacer y vivir en condiciones de alto riesgo, se desarrollan psicológicamente sanos y socialmente exitosos. Específicamente en el caso de la Psicología, la palabra no se limita a la resistencia, sino que conlleva un aspecto dinámico que implica que el individuo traumatizado se sobrepone (se desarrolla tras una pausa) y se reconstruye.

Para la palabra resiliencia, no existe aún un consenso sobre su definición, ya que son muchos los autores que incorporan el concepto; pero casi todas las que figuran en la bibliografía intentan explicarla en términos generales como: proceso, capacidad, crecimiento, enfrentamiento y habilidad.

Para esta investigación resiliencia se define como el resultado de la combinación y/o interacción entre los atributos del individuo (internos) y su ambiente familiar, social y cultural (externos) que lo posibilitan a superar el riesgo y la adversidad de forma constructiva ${ }^{(2)}$. Esta definición ha servido de guía y eje para comprender la resiliencia.
Por otro lado, cuando se está comprometida la salud, específicamente nos referimos al cáncer infantil, se tienen que proporcionar cuidados especiales, por lo que resulta ser una experiencia que representa un constante desafío que requiere la adaptación diaria del individuo; tanto del enfermo, como de la familia.

En México, el cáncer es la segunda causa más frecuente de mortalidad infantil en niños con edades entre 1 a 14 años. Las cifras oficiales del Instituto Nacional de Estadística Geografía e Informática $<$ INEGI $>$ reportan 2.650 muertes de niños con cáncer en 2005. En el 2008 se reportaron 5720 muertes de niños de 1 a 4 años por leucemia que corresponde al $3,3 \%$, entre los 5 a 14 años el porcentaje de mortalidad infantil se incrementa al 8,7 de un total de $6.585^{(3)}$.

En la mayoría de los casos se asume que el cáncer infantil es una experiencia traumática tanto para los niños como para sus familias, debido a las numerosas situaciones a las que tienen que enfrentarse en el transcurso de la enfermedad ya que representa una amenaza para la vida o integridad física. Lo anterior debido a los procedimientos médicos invasivos y dolorosos, tratamientos con efectos secundarios adversos, cambios físicos y funcionales, frecuentes hospitalizaciones, separación del grupo de iguales, alteración de la dinámica familiar, entre otros. Todas estas condiciones colocan a la población en riesgo de experimentar dificultades psicológicas a corto y largo plazo. Ya que se trata de una patología que puede curarse con el tiempo en algunos casos debido a los avances en los tratamientos, pero también puede tener consecuencias fatales para otros pacientes ${ }^{(4)}$.

El hecho de hacer frente a este problema de salud, significa confrontar trastornos físicos que de alguna forma pueden impactar en el aspecto psíquico de los individuos. $Y$ es cuando se inicia la 
tarea de aprender a manejar la enfermedad que corre más por cuenta del niño y de la familia. Además, como menciona Ortigosa, Méndez y Riquelme ${ }^{(5)}$, se espera tratar de ayudar al niño y a su familia a vivenciar la enfermedad con la menor afectación emocional y comportamental posible, así como minimizar el impacto psicológico que implica el diagnóstico y el tratamiento.

Autores como Keltner y Walter ${ }^{(6)}$, mencionan que no es una condición que una persona goce de buena salud para ser resiliente, sino que en muchos casos, el problema de salud, es el agente desencadenante de la resiliencia.

En este sentido, los planteamientos que surgen son: averiguar qué podrá ayudar al niño a superar unos años difíciles por motivo de la enfermedad y cómo conseguir que un niño que ha sufrido o está sufriendo situaciones duras, pueda ser capaz de aprender recursos que le ayuden tanto a soportar el golpe como a reestablecer su estabilidad emocional en ésta circunstancia, la cual, incluso es posible que se extienda en el tiempo. Las respuestas a estas interrogantes es que el niño necesita, además del tratamiento médico, de contar con resiliencia para construir nuevamente cimientos de estabilidad, y es a través de tutores afectivos, puntos de referencia, recuerdos de momentos felices y de personas que le han amado para que desde estas imágenes le ayuden a superar este tipo de situaciones ${ }^{(7,8)}$.

En el marco de la psicooncología pediátrica, se sugiere que los niños y adolescentes con cáncer afrontan la enfermedad y sus tratamientos con poco desgaste psicológico y que las alteraciones psicológicas o las disfunciones sociales son más la excepción que la regla, pues sólo afectan a un grupo reducido de niños, apreciándose cambios positivos en relación con el foco de las prioridades de la vida, comportamientos resilientes, más aprecio por la vida y las relaciones sociales así como menos conductas de riesgo que sus iguales, lo que desde el punto de vista de la salud constituye un excelente resultado(9).

Los estudios en resiliencia infanti $\left.\right|^{(2,10)}$ coinciden en indicar que la familia es un factor protector para el desarrollo de la resiliencia, y dentro de la familia, el principal rol promotor lo constituye la madre como cuidadora principal. Es así como la interacción funcional o disfuncional de la madre con el niño, genera en este último los aprendizajes que conformarán el tipo de vinculación afectiva y el estilo relacional de fortaleza o debilidad, que será la base para la actuación y respuestas del individuo ante los retos y demandas del entorno $^{(11)}$.

Partiendo de la idea de que particularmente es la madre quien principalmente es la encargada del cuidado del hijo, es posible pensar que puede influir sobre las características de éste, es decir, que ciertas conductas pueden afectar determinadas funciones infantiles ${ }^{(12)}$, pero también se puede suponer que ciertas conductas del infante pueden llegar a tener cierto impacto sobre la conducta materna. Por lo que a la fecha, parece que es importante seguir indagando la relación madre-hijo, como un elemento significativo en la evolución del niño y de otros procesos, para así comprender más acerca de la importancia que tiene en el desarrollo del pequeño.

$\mathrm{Al}$ respecto, se sabe que para que la resiliencia aflore en una situación de conflicto, es importante que desde la niñez los padres pongan atención en el desarroIlo de aptitudes sociales de sus hijos. Es a través de un apego seguro, el cual se forja en la relación con el otro (cuidador), mediante una interacción e intercambio que provee seguridad afectiva en los primeros años de vida y este tipo de interacción, especialmente la madre, se convierte en un mecanismo de protección ${ }^{(13)}$. Masten y Reed $^{(14)}$ describen que los padres que pro- 
mueven la resiliencia en sus hijos, se debe a que están involucrados en la educación de sus hijos, presentan conductas prosociales, de apoyo y comunicación con pares. Asimismo, Munist, Santos, Kotliarenco, Suárez, Infante y Grotberg ${ }^{(15-18)}$, coinciden en mencionar que el adulto que se interesa y proporciona estímulos, seguridad y una relación cariñosa, ayuda a determinar más que cualquier otro factor, la fuerza emocional en el niño, que a su vez repercutirá en su resiliencia.

El hecho de que un familiar esté enfermo, y más cuando se trata de un hijo, cambia todo, es una prueba muy costosa para los padres ${ }^{(7)}$, puesto que los tratamientos imponen un ritmo distinto para la familia y por supuesto un ajuste de roles, lo que requiere es que exista esta flexibilidad al interior de la familia para reubicarse en función del bienestar de todos los miembros.

Además, la presencia de un enfermo incita a un proceso de reflexión colectivo, Lea Baider ${ }^{(19)}$ explica que los miembros de la familia cercanos al paciente, con el que existe un enlace de cuidado y de ayuda mutua, se hacen parte integral en la trayectoria de la enfermedad, la cual afecta a cada miembro de la familia en diferentes áreas: emocionalmente, cognitivamente y en su conducta, en la rutina cotidiana, en los planes para el futuro, significado sobre uno mismo, sobre los demás e incluso al sentido de la vida. Asimismo, la enfermedad puede percibirse como un potencial peligro de desintegración de la familia, o bien como una oportunidad para el fortalecimiento de la misma, recuperación, adaptación y comprensión de las necesidades y expectativas de cada miembro familiar ${ }^{(14)}$.

Un meta-análisis de intervenciones psicológicas en enfermedades crónicas realizado por Martire, Lustig, Schulz, Miller y Helgeson ${ }^{(20)}$, procedieron a hacer una revisión de estudios elegidos comparan- do intervenciones dirigidas al paciente $y$ a miembros de la familia. En lo referente a los estudios dirigidos a los pacientes, las intervenciones tienen efectos positivos sobre la depresión cuando la pareja es incluida, incluso en los casos de pronóstico de muerte del paciente. Asimismo, han demostrado que los parientes cercanos de los pacientes experimentan pobre bienestar psicológico, disminuye la calidad de vida de la relación con el paciente y reducen su salud física(20,21).

Sin embargo, también es posible considerar que no todas las personas que cuidan de otros sufren estrés, pues parece que encontrar sentido a la situación que atraviesa un familiar con cáncer ayuda a una mejor adaptación a la situación y a un mayor bienestar de los cuidadores ${ }^{(22)}$. De tal forma que es importante que los estudios valoren tanto los aspectos positivos como negativos de la labor del cuidador y analicen la relación entre ellos para poder comprender mejor los efectos de esta compensación sobre el bienestar del cuidador ${ }^{(21)}$.

Los estudios sobre resiliencia apuntan a que se relaciona con muchas variables psicológicas, y son pocos los estudios que se han enfocado a estudiar la relación entre la resiliencia tanto de la madre como de sus hijos ${ }^{(4,23)}$. Por lo que se hace relevante conocer más acerca de las características de resiliencia entre madre e hijos. De tal forma que el objetivo de ésta investigación es, identificar características de resiliencia en madres y sus respectivos hijos, especialmente cuando estos tienen un diagnóstico de cáncer. Se espera evaluar los efectos en ambos miembros de la diada madre-hijo y no sólo uno de ellos. Al realizar una revisión respecto al enfermo oncológico, se encontró que incluyen a la familia o uno de sus miembros y se centran en la efectividad del tratamiento para mejorar diferentes síntomas o sufrimiento psicológico en los pacientes o sus 
familiares, reconociéndose así que ambos actores deben ser atendidos.

\section{MÉTODO}

\section{Participantes}

A través de un muestreo no probabilístico de tipo intencional, la muestra estuvo formada por un total de 60 participantes, de los cuales 30 son niños de ambos sexos (18 hombres, 12 mujeres), 29 casos con diagnóstico de Leucemia aguda linfoblástica y 1 caso con leucemia mieloide aguda y que actualmente están siendo sometidos a tratamiento en un Hospital público Infantil en la Ciudad de Toluca Estado de México; entre 8 y 17 años de edad $(X=13,13$; DS= $2,78)$. En lo referente al tiempo transcurrido desde el diagnóstico es muy variable por lo que no fue posible agruparlos, en cuanto a la fase de tratamiento un $90 \%$ se encuentran en mantenimiento, un caso abandonó el tratamiento y otro está en recaída. Respecto al tipo de tratamiento no se obtuvo el acceso a esta información. Los restantes participantes son sus respectivas madres, que se encuentran entre los 28 y 60 años de edad $(X=42,13, D S=$ $9,56)$, originarias de diferentes municipios y zona conurbada de la ciudad de Toluca Estado de México, con un nivel educativo de primaria hasta educación superior (ver tabla 1).

\section{Instrumentos}

Los instrumentos de medida de la resiliencia fueron dos:

1. Para los niños se aplicó el Cuestionario de Resiliencia ${ }^{(24)}$ que es un instrumento de autoinforme desarrollada en México con niños y adolescentes que mide factores específicos de la resiliencia. Consta de 32 reactivos de opciones de respuesta tipo Likert de cinco puntos (el valor 1 indica nunca y el 5 siempre). Del análisis factorial exploratorio con rotación ortogonal (varimax) y con base en el punto de inflexión de la curva de sedimentación, expectativas teóricas e interpretabilidad se optó por la solución de tres factores que explica una varianza acumulada de $37,82 \%$ y un COeficiente de confiabilidad a través de Alfa de Cronbach total $=0,9192$. Se considera la media teórica de 3. Está dividida en tres dimensiones que son:

- Factores protectores internos: denota funciones que se relacionan con habilidades para la solución de problemas (Alfa de Cronbach =0,8059, con 14 reactivos).

- Factores protectores externos. Se refiere a la posibilidad de contar con apoyo de la familia $\mathrm{y} / \mathrm{o}$ personas significativas para el individuo (Alfa de Cronbach $=0,7379$, con 11 reactivos).

- Empatía, denota comportamiento altruista y prosocial (Alfa de Cronbach $=0,7800$, con 7 reactivos).

La consistencia interna con los 32 reactivos para esta investigación es alta (Alfa de Cronbach total $=0,899$ ).

2. Para las mamás se les aplicó la Escala de Resiliencia (Fuerza y seguridad personal) para adultos de González Arratia et al., (25) consta de 50 reactivos con un rango de respuesta de diez puntos (0 al 10) para cada reactivo. Se tienen una varianza total explicada por los primeros cinco factores de $49,48 \%$ y confiabilidad satisfactoria (Alfa de Cronbach total=0,9065). Fue validada en adolescentes mexicanos (González Arratia et al., ${ }^{(25,26)}$ obteniéndose un coeficiente Alfa de Cronbach=0,92. En el caso de este estudio se obtuvo una confiabilidad a través de Alfa de Cronbach total $=0,940$.

Para su interpretación se consideran 3 niveles: 0 a 3 baja resiliencia, 4 a 7 moderada y de 8 a 10 alta resiliencia. Su estruc- 
Tabla 1. Características de la Muestra y Niveles de Resiliencia Madres e Hijos

\begin{tabular}{|c|c|c|c|c|c|c|c|}
\hline Caso & $\begin{array}{c}\text { Sexo } \\
\text { del } \\
\text { hijo }\end{array}$ & $\begin{array}{l}\text { Diagnós- } \\
\text { tico }\end{array}$ & $\begin{array}{r}\text { Edad } \\
\text { Del } \\
\text { hijo }\end{array}$ & $\begin{array}{c}\text { Nivel de } \\
\text { Resiliencia } \\
\text { hijo }\end{array}$ & $\begin{array}{c}\text { Escolaridad de la } \\
\text { madre }\end{array}$ & $\begin{array}{l}\text { Edad } \\
\text { de la } \\
\text { madre }\end{array}$ & $\begin{array}{c}\text { Nivel de } \\
\text { Resiliencia de } \\
\text { la madre }\end{array}$ \\
\hline 1 & $\mathrm{H}$ & $\mathrm{LAL}$ & 16 & Moderada & Primaria & 40 & Moderada \\
\hline 2 & M & $\mathrm{LAL}$ & 9 & Alta & Primaria incompleta & 35 & Moderada \\
\hline 3 & $\mathrm{H}$ & $\mathrm{LAL}$ & 11 & Moderada & Primaria incompleta & 45 & Baja \\
\hline 4 & $\mathrm{H}$ & $\mathrm{LAL}$ & 10 & Alta & Secundaria & 23 & Moderada \\
\hline 5 & $\mathrm{H}$ & LAL & 14 & Moderada & Secundaria & 37 & Moderada \\
\hline 6 & M & LAL & 17 & Alta & Primaria incompleta & 35 & Moderada \\
\hline 7 & M & LAL & 15 & Alta & No estudio & 36 & Moderada \\
\hline 8 & $\mathrm{H}$ & $\mathrm{LAL}$ & 14 & Moderada & Primaria & 55 & Baja \\
\hline 9 & $\mathrm{H}$ & $\mathrm{LAL}$ & 16 & Alta & Primaria & 42 & Moderada \\
\hline 10 & $\mathrm{H}$ & $\mathrm{LAL}$ & 17 & Alta & Primaria & 53 & Moderada \\
\hline 11 & M & $\mathrm{LAL}$ & 9 & Moderada & Primaria & 45 & Moderada \\
\hline 12 & $\mathrm{H}$ & $\mathrm{LAL}$ & 14 & Moderada & Primaria & 42 & Moderada \\
\hline 13 & M & $\mathrm{LAL}$ & 8 & Alta & Primaria incompleta & 54 & Moderada \\
\hline 14 & M & $\mathrm{LAL}$ & 15 & Alta & No estudio & 52 & Moderada \\
\hline 15 & M & LMA & 17 & Moderada & No estudio & 60 & Baja \\
\hline 16 & $\mathrm{H}$ & $\mathrm{LAL}$ & 12 & Alta & Primaria incompleta & 33 & Moderada \\
\hline 17 & $\mathrm{H}$ & $\mathrm{LAL}$ & 9 & Moderada & Primaria & 28 & Baja \\
\hline 18 & $\mathrm{H}$ & LAL & 16 & Alta & Primaria & 35 & Moderada \\
\hline 19 & $\mathrm{H}$ & LAL & 15 & Baja & Primaria incompleta & 50 & Moderada \\
\hline 20 & $\mathrm{H}$ & LAL & 15 & Alta & No estudio & 51 & Moderada \\
\hline 21 & $\mathrm{H}$ & $\mathrm{LAL}$ & 15 & Alta & Técnico & 39 & Moderada \\
\hline 22 & $\mathrm{H}$ & $\mathrm{LAL}$ & 8 & Alta & Preparatoria & 29 & Moderada \\
\hline 23 & M & $\mathrm{LAL}$ & 9 & Alta & Primaria & 43 & Moderada \\
\hline 24 & M & LAL & 12 & Alta & No estudio & 47 & Moderada \\
\hline 25 & $\mathrm{H}$ & $\mathrm{LAL}$ & 14 & Moderada & Primaria & 51 & Moderada \\
\hline 26 & $\mathrm{H}$ & $\mathrm{LAL}$ & 10 & Alta & Licenciatura & 50 & Moderada \\
\hline 27 & $\mathrm{H}$ & $\mathrm{LAL}$ & 9 & Alta & No estudio & 35 & Moderada \\
\hline 28 & M & LAL & 15 & Alta & Ingeniería & 39 & Baja \\
\hline 29 & M & $\mathrm{LAL}$ & 14 & Alta & Licenciatura & 55 & Moderada \\
\hline 30 & $M$ & LAL & 14 & Alta & No estudio & 29 & Moderada \\
\hline
\end{tabular}

Sexo: $\mathrm{H}=$ hombre, $M=$ mujer; Diagnóstico: $\mathrm{LAL}=$ leucemia aguda linfoblástica, $\mathrm{LMA}=$ leucemia mieloide aguda 
tura factorial indica que está compuesta por cinco factores que son:

- Seguridad personal. Indica juicios acerca de sí mismo, sobre la capacidad que tiene el individuo para la solución de problemas y genera confianza y sentido de competencia. Consta de 16 reactivos y un Alfa de Cronbach $=0,810$.

- Autoestima. Percepción de sí mismo de aceptación y aprecio. Consta de 20 reactivos (Alfa de Cronbach= 0,822).

- Afiliación. La percepción que tiene el individuo de que cuenta con redes de apoyo, genera un sentimiento de pertenencia, vínculos significativos para enfrentar una situación de adversidad. Consta de 5 reactivos (Alfa de Cronbach=0,816).

- Altruismo. Se refiere a juicios generales acerca de la capacidad de proveer ayuda a otros. Consta de 5 reactivos (Alfa de Cronbach= 0,520).

- Familia. Elemento protector que se constituye de vínculos afectivos que le ofrecen seguridad, estabilidad y apoyo proporcionado por al menos un adulto significativo. Consta de 4 reactivos (Alfa de Cronbach $=0,656$ ).

\section{Procedimiento}

Para seleccionar la muestra se acudió al hospital para solicitar los permisos respectivos. Los médicos fueron quienes indicaron quiénes eran los pacientes que podían participar en el estudio y que estaban en condiciones de responder a los cuestionarios. Todas las aplicaciones se realizaron de manera individual, tanto para los niños como para las mamas. En el caso de las mamás de los pacientes, además de explicar el objetivo, se les solicitó su consentimiento informado para la aplicación de la prueba a ellas y a sus respectivos hijos. La tasa de respuesta por parte de las madres fue de casi el 98 por ciento y el resto no desearon participar explicando no tener tiempo. En general el tiempo de aplicación fue de aproximadamente dos horas, considerándose las normas éticas del trabajo con personas.

\section{RESULTADOS}

Se hizo un análisis de $U$ de MannWhitney para observar si había diferencias en el cuestionario de resiliencia para niños de acuerdo con la variable sexo, en donde se encontró que no existen diferencias estadísticamente significativas en resiliencia entre niños y niñas ( $U$ de MannWhitney $=78,50, p=0,215$, niños rango= 13,86 , niñas rango $=17,96$, considerando una $p \leq 0,05)$. En la tabla 2 se muestra la media y desviación estándar obtenida en ambos sexos, la cual indica características de resiliencia superiores a la media teórica (3) en los factores protectores internos, externos y empatía en ambos sexos, y ligeramente superior en el caso de las niñas (Ver tabla 2).

Asimismo, se obtuvo la media y desviación estándar obtenidas para resiliencia de las mamás, en donde se encontró que entre sus características de resiliencia el altruismo se presenta en mayor medida, seguida de un nivel de resiliencia moderada en las dimensiones de: autoestima, familia, seguridad y por último afiliación (Ver tabla 3).

Se realizó un análisis de asociación entre las dimensiones del cuestionario de resiliencia del niño y de la madre, y en el análisis de Correlación de Spearman considerando una $p=0,05$, se encontró sólo una relación entre las variables, que indican que en la medida en que las mamás posean seguridad como parte de su resiliencia, mayor empatía por parte de su hijo (Rho de Spearman $=0,46, p=0,001$ ). En el 
Tabla 2. Media y Desviación estándar Resiliencia en Hijos

\begin{tabular}{|l|l|l|l|l|l|l|l|l|l|l|}
\hline Dimensión & $\begin{array}{c}\text { Media } \\
\text { Niños } \\
(\mathbf{n = 1 8})\end{array}$ & DE & Mínimo & Máximo & $\begin{array}{c}\text { Nivel } \\
\text { de } \\
\text { resiliencia }\end{array}$ & $\begin{array}{c}\text { Media } \\
\text { Niñas } \\
(\mathbf{n = 1 2})\end{array}$ & DE & Mínimo & Máximo & $\begin{array}{c}\text { Nivel } \\
\text { de } \\
\text { resiliencia }\end{array}$ \\
\hline $\begin{array}{l}\text { Factores } \\
\text { protectores } \\
\text { internos }\end{array}$ & 3,61 &, 68 & 1,64 & 4,64 & Alta & 3,98 &, 62 & 2,86 & 4,93 & Alta \\
\hline $\begin{array}{l}\text { Factores } \\
\text { protectores } \\
\text { externos }\end{array}$ & 4,11 &, 60 & 3,18 & 5,00 & Alta & 4,15 &, 62 & 3,00 & 5,00 & Alta \\
\hline Empatía & 3,70 &, 66 & 1,43 & 5,00 & Alta & 4,17 &, 62 & 2,86 & 5,0 & Alta \\
\hline
\end{tabular}

Tabla 3. Media y Desviación estándar Resiliencia en Madres.

\begin{tabular}{|c|c|c|c|}
\hline Dimensiones & $\begin{array}{c}\text { Media } \\
(\mathbf{n = 3 0})\end{array}$ & DE & Nivel de Resiliencia \\
\hline Seguridad & 7,00 & 1,87 & Moderada \\
\hline Autoestima & 7,32 & 1,96 & Moderada \\
\hline Afiliación & 6,73 & 2,53 & Moderada \\
\hline Altruismo & 8,72 & 1,65 & Alta \\
\hline Familia & 7,05 & 2,55 & Moderada \\
\hline
\end{tabular}

resto de las dimensiones no existe relación estadísticamente significativa.

\section{Discusión}

Los resultados de este trabajo sugieren que el nivel de resiliencia de los niños es alto, en los factores protectores internos, externos y empatía, lo cual apunta a considerar que el hecho de padecer una enfermedad como lo es el cáncer, están mostrando estas características favorables y que posiblemente les permiten y puede ayudar a dotar de sentido a esta experiencia así como dominar los desafíos que les impone la enfermedad.
Asimismo, los resultados indican un nivel de resiliencia de moderada a alta en el caso de las madres, lo cual, es un resultado alentador en el sentido de que en la medida que la madre posea mayor seguridad, autoestima, filiación, altruismo y considere a la familia como un apoyo en una situación como la de tener un hijo enfermo, pueden ser factores que ayuden a verse a sí mismas y a la relación con su hijo como algo más que el producto de la enfermedad $^{(10)}$.

Al relacionar las características de resiliencia de madre-hijo, se encontró sólo una asociación que indica que a mayor empatía por parte de los hijos, mayor al- 
truismo en las madres. Esto confirma la importancia de la familia y el papel de los padres en la educación de los hijos, para formar habilidades sociales que les permitan resolver los distintos problemas de la vida y que en el caso del niño enfermo y su madre tiene una relación recíproca. La empatía está asociada con habilidades sociales para experimentar y expresar tanto emociones positivas como negativas, y en el caso de este estudio se presentan ligeras diferencias a favor de las niñas como más empáticas, lo cual también es reportado por Marques y Koller ${ }^{(28)}$. En el caso del altruismo puede deberse que habitualmente la adversidad genera mayor empatía, altruismo y compasión por los otros, los mayores beneficios de la resiliencia ${ }^{(8)}$.

Hoy en día se reconoce la existencia de fuentes de apoyo en atención a la enfermedad, y es particularmente preponderante el papel de la familia y especialmente, es la madre quien asume esta responsabilidad del cuidado de los hijos y más aún cuando está enfermo. Por lo que, se considera que los servicios de salud podrán tener beneficios al incorporar a las madres como cuidadoras de sus hijos tanto que se ha demostrado que su participación ayuda a los objetivos programados, además de que la familia es una fuente disponible y accesible de apoyo para los enfermos.

Ver sufrir al ser más querido constituye a veces una prueba que parece imposible de superar y estos resultados dejan ver que en ambos casos, las madres y sus respectivos hijos, tienen la resistencia para avanzar en medio de la dificultad concreta que es la enfermedad. Ya que, es durante los peores tiempos cuando las personas intrínsecamente sobresalen por su capacidad de resolver problemas de manera creativa, sus expectativas positivas y su resiliencia ${ }^{(29)}$.

Por lo que se concluye que, si hay relación entre la resiliencia materna y la de su hijo, sin embargo, es importante destacar que como no se encontraron asociaciones en el resto de las dimensiones, resulta importante seguir probando ésta hipótesis a fin constatar ésta interacción recíproca. También entre las limitantes del presente estudio, es el número de participantes, por lo que se recomienda ampliar la muestra, así como el factor tiempo transcurrido a partir del diagnóstico, el hecho de que el niño estuviera sometido actualmente a tratamiento, o no lo hubiera completado, pueden ser variables importantes de considerar en futuros estudios, ya que posiblemente esto pueda tener un impacto diferencial en la resiliencia tanto del niño como de la madre.

Además, parece oportuno indicar que ante la enfermedad surgen respuestas tanto negativas como positivas por parte de los enfermos que las padecen. Por lo tanto, en parte, también puede resultar beneficioso que no solo se de tratamiento de tipo médico, sino que también se de intervención psicológica que incluya diversas áreas de actuación, entre otras, ayudar a los padres a manejar el estrés asociado al diagnóstico y al comienzo del tratamiento, instruirles para que sepan cómo apoyar a sus hijos durante el proceso y para que se reincorporen a su rutina cotidiana en las mejores condiciones posibles ${ }^{(9,21)}$.

Además, en el caso de las madres se hace imprescindible apoyarlas en términos de recalcar la importancia de su rol y en la necesidad de su presencia permanente, caracterizada por un contacto cercano, estimulador y efectivo para el niño ${ }^{(30)}$. Y esto a su vez es una necesidad de estudiar el efecto de los beneficios de las relaciones de apoyo en este tipo de enfermedad como lo es el cáncer infantil.

Finalmente, estos resultados llevan a considerar lo complejo que es el estudio de la resiliencia, y de reconocer la existencia de características resilientes en ambas partes y no considerar la situación como 
homogénea, sino que puede tener sus particularidades como edad de inicio de la enfermedad, tipo de relación entre madre e hijo y su asociación con otras variables como el estilo de afrontamiento. Lo cual nos abre un nuevo campo para la comprensión de la conducta resiliente de los individuos.

\section{REFERENCIAS BIBLIOGRÁFICAS}

1. Vázquez C. La ciencia del bienestar psicológico. En Vázquez, A. Hervás, G. Editores. La Ciencia del Bienestar. Madrid, España: Alianza Editorial; 2009; p. 13-43.

2. González-Arratia LFNI. Factores Determinantes de la Resiliencia en niños de la Ciudad de Toluca. [tesis doctoral]. Universidad Iberoamericana; 2007.

3. Instituto Nacional de Estadística Geografía e Informática. INEGI. [acceso 5 de septiembre 2010]. Disponible en: http://inegi.org. mx/. La Mortalidad infantil en México. Estimación por entidad Federativa 2006-2008.

4. Neil L, Clarke S. Learning to live with chilhood cancer: a literatura review of the parental perspective. Inter J Palliat Nurs 2010; 16(3): 110-9.

5. Ortigosa JM, Méndez FX, Riquelme A. Afrontamiento psicológico de los procedimientos médicos invasivos y dolorosos aplicados para el tratamiento del cáncer infantil y adolescente: La perspectiva cognitivoconductual. Psicooncología 2009; 6(2-3): 413-28.

6. Keltner B. Walter L. La resiliencia para aquellos que necesitan cuidados de la salud. En: Grotberg E.H. La resiliencia en el mundo de hoy. Barcelona España: Gedisa. 2006; p. 209-34.

7. Catret MA. Infancia y resiliencia. Actitudes y recursos ante el dolor. Valencia: Brief; 2007.

8. Grotberg, E.H. editor. La resiliencia en el mundo de hoy. Cómo superar las adversidades. Barcelona, España: Gedisa; 2006. Cap. 1, 8.
9. Bragado, C. Funcionamiento psicosocial e intervenciones psicológicas en niños con cáncer. Psicooncología 2009; 6(2-3): 32741.

10. Walsh, F. Resiliencia familiar. Buenos Aires, Argentina: Amorrortu, 2004.

11. Arana, A. Resiliencia: aprendiendo a sobreponerse a la tragedia y a la catástrofe personal. 2010. [acceso 23 de agosto 2010]. Disponible en: http://www.psicologia-online.com/

12. Segal J. Yahraes H. El crecimiento interior del niño. México: El Ateneo; 1982.

13. Cyrulnik B. La maravilla del dolor: el sentido de la resiliencia. España: Granica; 2001.

14. Garassini ME. Resiliencia y familiares de enfermos oncológicos. Universidad Metropolitana, Venezuela; 2010.

15. Munist M, Santos H, Kotliarenco MA, Suárez Ojeda EM, Infante F, Grotberg E. Manual de identificación y promoción de la resiliencia en niños y adolescentes. Fundación WK Kellogs Washington, D.C. 1998.

16. Bernard CP. Resiliency: A child in our perception? Am J Fam Ther 1994; 22: 135-44.

17. Vinson AJ. Children with Asthma: Initial development of child resilience model. Pediatr Nurs 2002; 28 (2): 149-58.

18. Rojas M. Factores de riesgo y protectores identificados en adolescentes consumidores de sustancias psicoactivas. Revisión y análisis del estado actual. 2007. [acceso 20 de abril de 2007]. Disponible en: http:// www.cedr.org.pe/ebooks/riesgo.

19. Baider L. Cáncer y familia: aspectos teóricos y terapéuticos. Rev Inter Psicol Clín Salud. 2003; 1(3): 505-20.

20. Martire LM. Lustig AP, Schulz R, Miller GE, Helgeson VS. Is it beneficial to involve a family member? A meta-analysis of psychosocial interventions for chronic illness. Health Psychol 2004; 23(6), 599-611. Doi:10.1037/0278-6133.23.6.599.

21. Morrison, V. Bennett, P. Psicología de la salud. España: Prentice Hall; 2007. 
22. López J, Rodríguez MI. La posibilidad de encontrar sentido en el cuidado de un ser querido con cáncer. Psicooncología 2007; 4, 1: 111-20.

23. Brennan P, Le Brocque R. Hammen C. Maternal depression parent-child relationships and resilient outcomes in adolescence. J Am Acad Child Adolesc Psychiatry 2003, 42(12): 1469-77. Doi:10.1097/00004583200312000-00014.

24. Levine KA. Against all odds: Resilience in single mothers of children with disabilities. Soc Work Health Care 2009; 48: 402-19. Doi:10.1080/00981380802605781.

25. González-Arratia, L.F.N.I., Valdez, M.J.L. De Jesús, S.M. Medición de la resiliencia, construcción y validación de una escala para niños. En: Sánchez AS, Díaz Loving R, Rivera AS. editores. México: La Psicología Social en México. 2006; Vol XI, (1). p 38690.
26. González-Arratia LFNI., Valdez, MJL. Zavala BY. Resiliencia en adolescentes mexicanos. Enseñ Invest Psicol 2008; 13(1): 41-52.

27. Villegas, S. E.G. Zamudio, P.M.C. Resiliencia. Hacia una validación de una escala en adolescentes. [tesis de licenciatura]. Universidad Autónoma de Yucatán. 2006.

28. Cecconello AM, Koller SH. Competência social e empatia: Um estudo sobre resiliência com criancas em situação de pobreza. Estud Psicol (Natal) 2000; 5(1): 71-93.

29. Siebert, A. La resiliencia. Construir en la adversidad. Barcelona, España: Alienta Editorial; 2007.

30. Kotliarenco, M.A., Cáceres, I. Fontecilla, M. Estado del arte en resiliencia. 1997. Washington D.C., Organización Panamericana de la Salud. [acceso 20 de agosto de 2003]. Disponible en: http://www.resiliencia.cl/investig/index.htm. 
